\title{
To screen or not to screen? Is it worth it for oral cancer?
}

\section{Is a primary care screening programme for oral cancer cost-effective?}

Speight PM, Palmer S, Moles DR, et al.

The cost-effectiveness of screening for oral cancer in primary care. Health Technol Assess 2006; 10(14).

Design The cost-effectiveness of oral cancer screening programmes in a number of primary care environments was simulated using a decision analysis model. Primary data on actual resource use and costs were collected by case note review in two hospitals. Additional data needed to inform the model were obtained from published costs, from systematic reviews and by expert opinion using the Trial Roulette approach ${ }^{1}$. The value of future research was determined using 'expected value of perfect information' (EVPI) for the decision to screen and for each of the model inputs.

Setting Hypothetical screening programmes conducted in a number of primary care settings. Eight strategies were compared: (A) no screen; (B) invitational screen in general medical practice; (C) invitational screen in general dental practice; (D) opportunistic screen in general medical practice; (E) opportunistic screen in general dental practice; (F) opportunistic high-risk screen in general medical practice; (G) opportunistic high-risk screen in general dental practice; and $(\mathrm{H})$ invitational screen with a specialist. Participants were a hypothetical population over the age of 40 years of age.

Main outcome measures The main measures were mean lifetime costs and quality-adjusted life-years (QALY) of each alternative screening scenario and incremental cost-effectiveness ratios (ICER) to determine the additional costs and benefits of each strategy over another.

Results No screening (strategy $A$ ) was always the cheapest option. Strategies B, C, E and $\mathrm{H}$ were never cost-effective and were ruled out by dominance or extended dominance. Of the remaining strategies, the ICER for the whole population (age 49-79 years) ranged from $£ 15790$ to $£ 25961$ per QALY. Modelling a $20 \%$ reduction in disease progression always gave the lowest ICER. Cost-effectiveness acceptability curves showed that there is considerable uncertainty in the optimal decision identified by the ICER, depending on both the maximum amount that the UK National Health Service may be prepared to pay and the impact that treatment has on the annual malignancy transformation rate. Overall, however, high-risk opportunistic screening by a general dental or medical practitioner (strategies $\mathrm{F}$ and $\mathrm{G}$ ) may be cost-effective. EVPI were high for all parameters with population values ranging from $£ 8$ million to $£ 462$ million. The values were significantly higher in males than females, however, but also varied depending upon malignant transformation rate, effects of treatment and willingness to pay. Partial EVPI showed the highest values for malignant transformation rate, disease progression, self-referral and costs of cancer treatment.

Address for correspondence: Professor P Speight, Department of Oral Pathology, School of Clinical Dentistry, University of Sheffield, Claremont Crescent, Sheffield S10 2TA, UK.
Conclusions Opportunistic high-risk screening, particularly in general dental practice, may be cost-effective. This screening may be more effectively targeted to younger age groups, particularly 40-60 year olds. There is considerable uncertainty in the parameters used in the model, however, particularly malignant transformation rate, disease progression, patterns of self-referral and costs. Further study is needed on malignant transformation rates of oral, potentially malignant lesions and to determine the outcome of treatment of those lesions. Evidence has been published to suggest that intervention has no greater benefit than 'watch and wait' and, hence, a properly planned randomised controlled trial may be justified. Research is also needed into the rates of progression of oral cancer and on referral pathways from primary to secondary care and their effects on delay and stage of presentation.

\section{Commentary}

Oral cancer incidence continues to rise across the $\mathrm{UK}^{2}$ and survival rates have not improved significantly in the last 30 years. ${ }^{3}$ In an attempt to improve this situation, oral cancer screening — basically early detection and intervention - is an instinctively attractive idea.

Criteria have been developed to aid decisions about whether a screening programme is worthwhile. These include determining the evidence base, and assessing the benefits versus harms, with the measurement of cost-effectiveness a key question. ${ }^{4}$ The primary aim of this report was to address the question of cost-effectiveness for oral cancer. A range of options for oral cancer screening programmes (including no screening, invitational screening, opportunistic screening and targeted screening) in a range of primary care settings by general medical practitioner, general dental practitioner, or specialist were explored.

Within the report, a systematic review of the sensitivity and specificity of oral cancer screening found generally high test performance, but no additional benefit was found with the use of toluidine blue staining. A second systematic review of the evidence on the potential health benefits associated with oral cancer screening was equivocal. Resources and costs for management of oral cancer and precancer in primary care and secondary care were determined via questionnaires, expert opinion and case-note review in two hospitals. A third systematic review of the literature on the cost-effectiveness of screening for oral cancer disappointingly yielded only one study with a full economic evaluation. This latter finding that there is a paucity of evidence limited the comparison of alternative screening strategies from the data that do exist.

The authors created a cost-effective analytical decision model populated with the evidence they were able to compile. The main findings are presented with a degree of caution, resulting from the uncertainty of the data in the model parameters. It was shown that opportunistic targeted screening to high-risk groups, particularly in general dental practice, may be cost-effective.

Other questions were impossible to answer with current knowledge, however. These remaining uncertainties were listed, including: the malignant transformation rate, disease progression, patterns of service access and referral, and the full costs involved. Thus, the jury remains out on the core issue of cost-effectiveness of oral cancer screening. Further, a crucial issue, not addressed in this report, but important when considering a screening programme, is the broader 
economic issue of finite resources. This necessitates consideration of the cost-effectiveness of oral cancer screening relative to other health interventions which could be offered instead. ${ }^{4}$ Nevertheless, this report is a comprehensive gauge of the current research on oral cancer screening. Recommendations for further research are detailed and researchers in the field should employ this as the new baseline on the subject of oral cancer screening.

\section{Practice point}

From the evidence available in this review: general dental practitioners are in the best position to perform an oral screening for oral cancer and potentially malignant lesions. This should be incorporated into the general oral health history and examination and form a routine part of an oral cancer risk assessment which should also include enquiry regarding oral cancer risk factors.

\section{David I Conway}

Department of Dental Public Health, University of Glasgow

Dental School, Glasgow, Scotland, UK
1. Gore D. Biostatistics and the Medical Research Council. Med Res Council News 1987;36:19-20

2. Conway DI, Stockton DL, Warnakulasuriya KAAS, Ogden G, Macpherson LMD. Incidence of oral and oro-pharyngeal cancer in United Kingdom (1990-1999) - recent trends and regional variation. Oral Oncol 2006; 42:586-592.

3. Hindle I, Downer MC, Speight PM. Oral cancer in England and Wales 1901-1990. Br J Oral Maxillofac Surg 1996; 34:471-476.

4. Barratt A, Irwig L, Glasziou P, Cumming RG, Raffle A, Hicks N, et al.Users' guides to the medical literature. XVII. How to use guidelines and recommendations about screening. J Am Med Assoc 1999; 281:2029-2034.

5. Baum M. Screening for breast cancer, time to think — and stop? Lancet $1995 ; 346: 436$ Evidence-Based Dentistry (2006) 7, 81-82.

doi:10.1038/sj.ebd.6400435

Mouth Cancer Awareness Week will be running from Sunday 12 November to Saturday 18 November 2006. To end the Week the Mouth Cancer Foundation is organising a sponsored 10K Walk on Sunday 19 November in Hyde Park, London, at 10am For more information about the walk please see the website at www.mouthcancerwalk.org

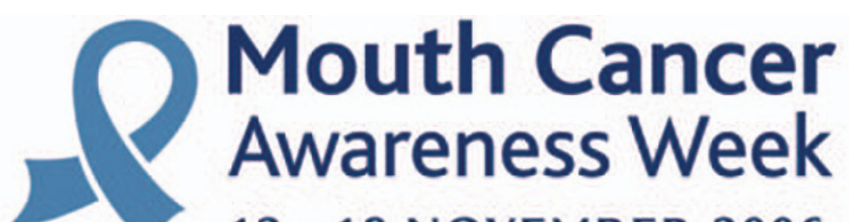
12 - 18 NOVEMBER 2006

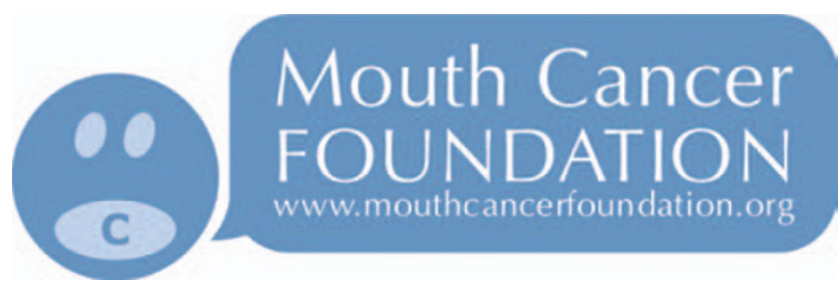

